\title{
A prospective, randomised, double blind placebo-controlled trial to evaluate the efficacy and safety of tocilizumab in patients with severe COVID-19 pneumonia (TOC-COVID): A structured summary of a study protocol for a randomised controlled trial
}

Jonathan Rilinger ${ }^{1,2^{*}}$ D. Winfried V. Kern ${ }^{3}$, Daniel Duerschmied ${ }^{1,2}$, Alexander Supady ${ }^{1,2}$, Christoph Bode ${ }^{1,2}$, Dawid L. Staudacher ${ }^{1,2}$ and Tobias Wengenmayer ${ }^{1,2}$

\footnotetext{
Abstract severe COVID-19. result in fewer admissions to intensive care units. influence the patient's outcome.

* Correspondence: jonathan.rilinger@uniklinik-freiburg.de

'Department of Medicine III (Interdisciplinary Medical Intensive Care), Medical

Center, University of Freiburg, Faculty of Medicine, University of Freiburg, Freiburg, Hugstetterstr. 55, 79106 Freiburg, Germany

${ }^{2}$ Department of Cardiology and Angiology I, Heart Center Freiburg University, Faculty of Medicine, University of Freiburg, Freiburg, Germany Full list of author information is available at the end of the article
}

Objectives: SARS-CoV2 infection leads to a concomitant pulmonary inflammation. This inflammation is supposed to be the main driver in the pathogenesis of lung failure (Acute Respiratory Distress Syndrome) in COVID-19. Objective of this study is to evaluate the efficacy and safety of a single dose treatment with Tocilizumab in patients with

We hypothesize that Tocilizumab slows down the progression of SARS-CoV-2 induced pneumonia and inflammation. We expect an improvement in pulmonary function compared to placebo-treated patients. Desirable outcomes would be that tocilizumab reduces the number of days that patients are dependent on mechanical ventilation and reduces the invasiveness of breathing assistance. Furthermore, this treatment might

Next to these efficacy parameters, safety of a therapy with Tocilizumab in COVID-19 patients has to be monitored closely, since immunosuppression could lead to an increased rate of bacterial infections, which could negatively

C The Author(s). 2020 Open Access This article is licensed under a Creative Commons Attribution 4.0 International License, which permits use, sharing, adaptation, distribution and reproduction in any medium or format, as long as you give appropriate credit to the original author(s) and the source, provide a link to the Creative Commons licence, and indicate if changes were made. The images or other third party material in this article are included in the article's Creative Commons licence, unless indicated otherwise in a credit line to the material. If material is not included in the article's Creative Commons licence and your intended use is not permitted by statutory regulation or exceeds the permitted use, you will need to obtain permission directly from the copyright holder. To view a copy of this licence, visit http://creativecommons.org/licenses/by/4.0/. The Creative Commons Public Domain Dedication waiver (http://creativecommons.org/publicdomain/zero/1.0/) applies to the data made available in this article, unless otherwise stated in a credit line to the data. 
(Continued from previous page)

Trial design: Multicentre, prospective, 2-arm randomised (ratio 1:1), double blind, placebo-controlled trial with parallel group design.

Participants: Inclusion criteria

1. Proof of SARS-CoV2 (Symptoms and positive polymerase chain reaction (PCR))

2. Severe respiratory failure:

a. Ambient air $\mathrm{SpO}_{2} \leq 92 \%$ or

b. Need of $\geq 6102 / \mathrm{min}$ or

c. NIV (non-invasive ventilation) or

d. IMV (invasive mechanical ventilation)

3. Age $\geq 18$ years

Exclusion criteria

1. Non-invasive or invasive mechanical ventilation $\geq 48$ hours

2. Pregnancy or breast feeding

3. Liver injury or failure (AST/ALT $\geq 5 \times$ ULN)

4. Leukocytes $<2 \times 10^{3} / \mu \mathrm{l}$

5. Thrombocytes $<50 \times 10^{3} / \mu \mathrm{l}$

6. Severe bacterial infection (PCT $>3 \mathrm{ng} / \mathrm{ml}$ )

7. Acute or chronic diverticulitis

8. Immunosuppressive therapy (e.g. mycophenolate, azathioprine, methotrexate, biologicals, prednisolone $>10 \mathrm{mg} / \mathrm{d}$; exceptions are: prednisolone $\leq 10 \mathrm{mg} / \mathrm{d}$, sulfasalazine or hydroxychloroquine)

9. Known active or chronic tuberculosis

10. Known active or chronic viral hepatitis

11. Known allergic reactions to tocilizumab or its ingredients

12. Life expectation of less than 1 year (independent of COVID-19)

13. Participation in any other interventional clinical trial within the last 30 days before the start of this trial 14. Simultaneous participation in other interventional trials (except for participation in COVID-19 trials) which could interfere with this trial; simultaneous participation in registry and diagnostic trials is allowed

15. Failure to use one of the following safe methods of contraception: female condoms, diaphragm or coil, each used in combination with spermicides; intra-uterine device; hormonal contraception in combination with a mechanical method of contraception.

The data collection of the primary follow up (28 days after randomisation) takes place during the hospital stay. Subsequently, a telephone interview on the quality of life is conducted after 6 and 12 months. Participants will be recruited from inpatients at ten medical centres in Germany.

Intervention and comparator: Intervention arm: Application of 8mg/kg body weight (BW) Tocilizumab i.v. once immediately after randomisation $(12 \mathrm{mg} / \mathrm{kg}$ for patients with <30 kg BW; total dose should not exceed $800 \mathrm{mg}$ ) AND conventional treatment. Control arm: Placebo (NaCl) i.v. once immediately after randomisation AND conventional treatment.

Main outcomes: Primary endpoint is the number of ventilator free days (d) (VFD) in the first 28 days after randomisation. Non-invasive ventilation (NIV), Invasive mechanical ventilation (IMV) and extracorporeal membrane oxygenation (ECMO) are defined as ventilator days. VFD's are counted as zero if the patient dies within the first 28 days.

(Continued on next page) 
(Continued from previous page)

Randomisation: The randomisation code will be generated by the CTU (Clinical Trials Unit, ZKS Freiburg) using the following procedure to ensure that treatment assignment is unbiased and concealed from patients and investigator staff. Randomisation will be stratified by centre and will be performed in blocks of variable length in a ratio of 1:1 within each centre. The block lengths will be documented separately and will not be disclosed to the investigators. The randomisation code will be produced by validated programs based on the Statistical Analysis System (SAS).

Blinding (masking): Participants, caregivers, and the study team assessing the outcomes are blinded to group assignment.

Numbers to be randomised (sample size): 100 participants will be randomised to each group (thus 200 participants in total).

Trial Status: Protocol Version: V 1.2, 16.04.2020. Recruitment began 27th April 2020 and is anticipated to be completed by December 2020.

Trial registration: The trial was registered before trial start in trial registries (EudraCT: No. 2020-001408-41, registered 21st April 2020, and DRKS: No. DRKS00021238, registered 22nd April 2020).

Full protocol: The full protocol is attached as an additional file, accessible from the Trials website (Additional file 1). In the interest in expediting dissemination of this material, the familiar formatting has been eliminated; this Letter serves as a summary of the key elements of the full protocol.

Keywords: COVID-19, Randomised controlled trial, protocol, Tocilizumab, IL-6-Rezeptor blockade, Ventilator free days, Inflammation, Pneumonia, SARS-CoV2

\section{Supplementary information}

Supplementary information accompanies this paper at https://doi.org/10. 1186/s13063-020-04447-3.

Additional file 1. Full study protocol.

\section{Acknowledgements}

Not applicable.

\section{Authors' contributions}

JR, WVK, DD, AS, CB, DLS and TW contributed to the conception of the study; JR, DLS and TW contributed to the preparation of the study protocol; JR, DLS and TW contributed to study organisation and obtaining approval of leading ethics committee and responsible competent authority; TW is Coordinating Investigator of this study; DLS and JR are Deputy coordinating Investigators of this study; JR and TW drafted the manuscript; WVK, DD, AS, $C B, D L S$ revised the manuscript for important intellectual content. All authors approved the final version of the manuscript.

\section{Funding}

Funding for this project has been requested at the German Federal Ministry of Education and Research (BMBF - Bundesministerium für Bildung und Forschung). The funding does and will not have an influence on the design of the study, collection, analysis and interpretation of data and in writing the manuscript besides financial support.

\section{Availability of data and materials}

Data will be available from the coordinating investigator of this study on reasonable request:

Tobias Wengenmayer

tobias.wengenmayer@uniklinik-freiburg.de

Department of Medicine III (Interdisciplinary Medical Intensive Care), Medical Center, University of Freiburg, Hugstetterstr. 55, 79106 Freiburg, Germany.

Tel: +49 761-270-34010; Fax: +49 761-270-963-3322

\section{Ethics approval and consent to participate}

Ethics committee of the Albert-Ludwigs-University Freiburg, Germany Ethical approval number: 203/20 (FF/MC), date of approval: 21.04.2020. We certify that this trial has received ethical approval from the appropriate ethical committee as described above.
Written informed consent will be obtained from the patient or legal authorized representative.

If patients are unable to consent because of a severe COVID-19 pneumonia and an official legal representative was not declared so far, patients can be enrolled when a participation conforms to the presumed will of the patient, according to international guidelines and local laws (§ 41 (1) sentence 2 and sentence $3 \mathrm{AMG}$ ).

\section{Consent for publication}

Not applicable.

\section{Competing interests}

The authors declare that they have no competing interests.

\section{Author details}

${ }^{1}$ Department of Medicine III (Interdisciplinary Medical Intensive Care), Medical Center, University of Freiburg, Faculty of Medicine, University of Freiburg, Freiburg, Hugstetterstr. 55, 79106 Freiburg, Germany. ${ }^{2}$ Department of Cardiology and Angiology I, Heart Center Freiburg University, Faculty of Medicine, University of Freiburg, Freiburg, Germany. ${ }^{3}$ Division of Infectious Diseases, Department of Medicine II, Medical Center - University of Freiburg, Faculty of Medicine, University of Freiburg, Freiburg, Germany.

Received: 20 May 2020 Accepted: 23 May 2020

Published online: 03 June 2020

\section{Publisher's Note}

Springer Nature remains neutral with regard to jurisdictional claims in published maps and institutional affiliations. 